\title{
PARTICIPACIÓN FEMENINA EN GESTIÓN DIRECTIVA UNIVERSITARIA EN CHILE
}

FEMALE PARTICIPATION IN UNIVERSITY MANAGEMENT IN CHILE

\author{
Benjamín Hirsch Gómez
}

\begin{abstract}
Resumen
Esta investigación tiene por objeto caracterizar la participación de mujeres en cargos directivos de gestión universitaria, específicamente en los cargos unipersonales de rectoría, vicerrectoría y decanatos de las universidades en Chile. Esto, con el propósito de analizar las barreras y limitantes que se les presentan a las mujeres para posicionarse laboralmente y que explican la baja presencia de estas en cargos directivos, con una perspectiva a futuro que permita un avance de la inserción laboral femenina en la gestión universitaria.

Esta investigación posee un carácter mixto de naturaleza tanto cuantitativa como cualitativa. Para este efecto se realiza una investigación y análisis de fuentes primarias, a través de páginas web de las universidades que forman parte de la muestra y se complementa el análisis con la opinión de informantes claves, a través de once entrevistas semiestructuradas.

Los resultados de la investigación principalmente concluyen que en Chile existe una baja participación femenina en los cargos de rectoría, vicerrectoría y decanatos, y que las principales barreras encontradas son: la incompatibilidad de responsabilidades familiares y laborales, la percepción de rechazo masculino y la naturaleza de las actividades académicas.
\end{abstract}

Palabras claves: gestión directiva universitaria, techo de cristal, equidad de género, barreras al desarrollo laboral femenino.

\begin{abstract}
:
The purpose of this research is to characterize women's participation in university management leadership positions, specifically in unipersonal positions such as Dean, Rector, Chancellor, Vice-Rector, and Vice-Chancellor of universities in Chile. This, with the purpose of analyzing the barriers and limitations that are presented to women to move up in the workplace and that explain the low presence of these in managerial positions, with a future perspective that allows an advance of female labor insertion in university management.

This research has a mixed character, both quantitative and qualitative in nature. For this purpose, an investigation and analysis of primary sources is carried out, through universities' web pages that are part of the sample. Eleven semi-structured interviews to key informants complement the analysis.

The results of the research mainly conclude that there is low female participation in Rectory, Vice-Rectory and Deanship positions, and that the main barriers found are: the incompatibility of family and work responsibilities, perception of male rejection and the nature of academic activities.
\end{abstract}

Keywords: university management, glass ceiling, gender equality, barriers to female labor development.

Recibido: 26 de octubre de 2021 /Aceptado: 18 de enero de 2022

\footnotetext{
*Administrador Público con Mención en Gestión Pública. Correo: benjahirsch23@gmail.com, ORCID: https://orcid.org/0000-0001$\underline{9168-7060}$
} 


\section{Introducción}

En la actualidad, la desigualdad de género es entendida como un problema público, siendo parte de la agenda pública estatal, esto debido principalmente a los deficientes datos de participación laboral femenina. Con la pandemia mundial de Covid19 estos datos han empeorado, en la actualidad la tasa de desocupación femenina en el trimestre enero-marzo 2021, alcanzó un 11,0\%, (INE, 2021), mientras que la tasa de participación laboral femenina en el 2020 fue de 45,3\%, convirtiéndose en la más baja desde el año 2010 (INE, 2020).

Además, el área gerencial y de toma de decisiones, sigue siendo un área muy masculina, en donde las mujeres son muy pocas. Según datos del Informe de Mujeres en Alta Dirección de Empresas (IMAD 2017) en 2017 sólo un 13\% de los cargos ejecutivos en el país era ocupado por mujeres. Esta cifra aumentó a un $18 \%$ en 2019 y en un $21 \%$ en el 2020, sin embargo, sigue siendo muy baja y sigue preocupando (IMAD, 2020).

Diversos estudios sobre participación femenina en educación superior, reconocen que esta problemática también se encuentra presente en las universidades.

La participación de estudiantes mujeres en la educación superior en los últimos años ha alcanzado niveles mayores al de los hombres (Del Pino Arriagada et al., 2018; Guil, 2014; Lorenzo, Sola y Cáceres, 2007), sin embargo, al ascender dentro de la organización, la participación femenina se ve disminuida, situación que se presenta en Chile y también a nivel internacional. Esto lo demuestran diversos estudios sobre la participación de la mujer en la educación superior, donde se reconoce que existen avances, no obstante, siguen persistiendo dificultades para que las mujeres accedan a puestos estratégicos en la toma de decisiones y en los puestos de poder. Mientras más alto el nivel jerárquico en la institución de educación, menor es la presencia de mujeres en cargos de responsabilidad superior (Del Pino Arriagada et al., 2018; Cárdenas, 2013; Gaete-Quezada, 2015).

Estas son algunas de las claves de la temática que se pretende analizar en esta investigación, el por qué este favorable aumento en la cantidad de mujeres que ingresan a estudiar a la universidad, no se traduce en un aumento en la cantidad de mujeres que trabajan en la gestión directiva universitaria.

Sobre la base del reconocimiento de estas problemáticas, esta investigación pretende identificar los factores y principales barreras que influyen en la baja participación de las mujeres en cargos de gestión directiva universitaria, específicamente en los cargos de rectoría, vicerrectoría y decanaturas.

\subsection{Diferenciación entre conceptos de Sexo y Género}

Es importante comenzar por diferenciar entre los conceptos de sexo y género, en donde el concepto de sexo hace alusión a las diferencias biológicas entre hombres y mujeres, mientras que el concepto de genero se refiere a "las diferencias sociales que son aprendidas culturalmente, variables en el tiempo y pueden manifestarse de distintas formas en distintas culturas" (Robert Stoller, 1968), definición de Robert Stoller quien fue uno de los pioneros en utilizar el concepto género en el ámbito de la psiquiatría. En las ciencias sociales la autora Rosa Cobo establece que el género "es una construcción cultural prescriptiva que se ha ido redefiniendo históricamente en función de la correlación de fuerzas de las mujeres en las distintas sociedades en que el feminismo ha arraigado social y culturalmente" (Cobo, 2005). Otra definición es la de Barberá que establece que "el género es un concepto dinámico y susceptible de variaciones, tanto de carácter temporal como situacionales e interindividuales” (Barberá, 1998). 
Posteriormente, los estudios feministas, con desarrollos conceptuales desde la sociología, la antropología, la historia, la filosofía, son los que definen el género como un "sistema de jerarquías sociales" que permiten problematizar la desigualdad de género. Desde ahí llega ste concepto al campo de la ciencia política y la administración pública. Por ende, el género no es estático, es dinámico, ya que éste depende del entorno social y cultural en el que una persona se desenvuelve (Cirillo, 2005, Mead, 1972).

\subsection{Igualdad de Género y su importancia}

Otro concepto importante es la igualdad de género, que es entendida como "la existencia de una igualdad de oportunidades y de derechos entre las mujeres y los hombres en las esferas privada y pública” (UNESCO, 2014).

La igualdad de género en la actualidad, constituye uno de los 17 objetivos de desarrollo sostenible de las Naciones Unidas. Y su importancia surge debido a que las mujeres representan la mitad de la población mundial, por lo que también representan la mitad de su potencial, por lo cual la desigualdad de género provoca el estancamiento del progreso social (PNUD, 2016). Además, el Programa de las Naciones Unidas para el Desarrollo destaca que el empoderar a las mujeres ayuda a promover el crecimiento económico y el desarrollo en el mundo (PNUD, 2016).

\subsection{Desigualdades de género}

Estas diferencias entre los conceptos de sexo y de género son estudiados por diversos autores, entre ellos Marta Lamas (1986) quien establece que estas diferencias entre hombres y mujeres se producen debido a una construcción cultural, la cual ha definido roles, actividades, tareas y responsabilidades distintas para hombres y mujeres. Estos roles son enseñados desde la infancia hacia niños y niñas y van guiando sus vidas (Lamas, 1986).

El estudio de estas diferencias, revela las desigualdades y discriminación que existe hacia las mujeres, principalmente en el área laboral. En esta área, se ha generado culturalmente e históricamente una división sexual del trabajo, la cual es un mandato social que atribuye a las mujeres trabajos domésticos y un rol en el área familiar, que les dificulta el ingreso al campo laboral, que es preponderantemente masculino (Astelarra, 2005).

Esta división sexual del trabajo genera la creencia de que existen trabajos adecuados y otros no adecuados para las mujeres, promoviendo el acceso de mujeres exclusivamente a aquellas posiciones congruentes con una imagen naturalizada de lo femenino (Eagly, 1987).

Estas diferencias culturales son explicadas por algunos autores, entre ellos Parson y Bales, que en 1955 identificaron dos características diferentes: a los hombres se les asigna un papel instrumental orientado a metas, mientras que a las mujeres se les asigna un papel expresivo orientado a las relaciones interpersonales (Lupano y Castro, 2010; Pearson, Bales, 1955). En la misma línea, Bakan (1966) distingue dos tipos de rasgos más comunes en hombres y mujeres: los rasgos de diligencia y de comunión. En los hombres, los más comunes son los rasgos de diligencia, caracterizados por la autoafirmación, la autoprotección, la competitividad, el control y la autoridad. Mientras que en las mujeres los rasgos de comunión o expresivos, se caracterizan por la abnegación y la preocupación por los demás (Lupano y Castro, 2010; Eagly 1987; Bakan, 1966).

Estas características culturalmente asociadas a hombres y mujeres, generan estereotipos de género, que relacionan rasgos masculinos con roles de liderazgo, lo que 
conlleva a que las mujeres no sean consideradas para estos cargos, fomentando sesgos contra las mujeres en ocupación de puestos directivos (Lupano y Castro, 2010; Cuadrado, 2004; Eagly 1987).

Esta división sexual del trabajo se ha ido rompiendo paulatinamente con el ingreso de las mujeres en el área laboral, debido al entendimiento de estas desigualdades como un problema y también debido al actuar de la comunidad internacional a través de tratados en favor de los derechos de la mujer.

\subsection{Teoría del Techo de Cristal}

Otro punto importante para entender esta problemática, es la teoría del "techo de cristal", la cual es una de las teorías más importantes y con más repercusión, en que los autores Hymowitz y Schelhardt en el año 1986, utilizan la expresión "techo de cristal" refiriéndose de manera metafórica a las barreras invisibles y estereotipos que afectan directamente a las mujeres y las frustra de tener un campo abierto para ascender hacia rangos de mayor jerarquía (U.S. Glass Ceiling Commission, 1995).

Es un fenómeno invisible encubierto, que no se expresa en ningún manual, ni en ninguna reunión de negocios y que deja las posiciones de liderazgo exclusivamente en manos de los hombres (Hymowitz, Schelhardt, 1986).

Esta expresión ha sido utilizada por diversos autores de manera de ilustrar las barreras que impiden que las mujeres asciendan y tengan un desarrollo progresivo en las empresas u organizaciones de manera igualitaria a los hombres.

\subsection{Techo de cristal en las Universidades}

Diversos diagnósticos y estudios sobre la participación de la mujer en la educación superior establecen que el techo de cristal también se manifiesta en las universidades. Sin embargo, una de las diferencias que supone el techo de cristal en las universidades con otras organizaciones del área laboral, es la existencia de un "mundo académico" el cual es estudiado por diversos autores.

Uno de estos autores es R.K. Merton quien, en el año 1968, explica este fenómeno dentro de las universidades, a través de la teoría del "Efecto Mateo". Merton utiliza una analogía bíblica del evangelio de Mateo: "porque al que tiene se le dará más y tendrá en abundancia, pero al que no tiene, se le quitará aun lo que tiene". Con esta analogía, Merton (1968) muestra lo jerarquizado que es el mundo académico, generando disputas entre los investigadores por mayor reconocimiento, prestigio, influencia y recursos (Merton, 1968; Becher, 2001; Gaete-Quezada, 2015).

La historiadora Margaret Rossiter, basándose en el efecto Mateo, propone la teoría del efecto Matilda, la cual explica que las mujeres son más vulnerables al efecto Mateo y que las recompensas en las ciencias son distribuidas de manera discriminatoria hacia las mujeres, generando un perjuicio en contra del reconocimiento hacia los logros científicos de las mujeres (Rossiter, 1993).

La UNESCO en su informe "Mujeres en la educación superior: ¿la ventaja femenina ha puesto fin a las desigualdades de género?" del 2021, concluye que "las mujeres están sobrerrepresentadas entre el personal docente de los niveles educativos inferiores, mientras que su presencia es notablemente menor en la enseñanza superior (segregación vertical). En muchos países, las mujeres siguen estando infrarrepresentadas en los niveles superiores del profesorado y en los órganos de decisión de la enseñanza superior" (UNESCO, 2021). 
Con respecto a estudios más específicos sobre participación femenina en gestión directiva universitaria en Chile, se pueden enumerar varios ejemplos:

En 2007, Berríos revela que un $23 \%$ de los cargos directivos superiores en universidades chilenas eran ejercidos por mujeres y que solo el $3 \%$ ejercía el cargo de rectora (Berríos, 2007).

En el año 2013, Cárdenas constata que en las universidades chilenas solo el 5,1\% son rectoras, mientras el 22,6\% son decanas o jefas de carrera (Cárdenas, 2013).

En el año 2015, Gaete-Quezada constata que el $100 \%$ de las universidades estatales chilenas posee como rector a un hombre (Gaete-Quezada, 2015).

Otro estudio del año 2016 pudo establecer que un 24,3\% de los cargos de alta dirección en las universidades estatales chilenas son ocupados por mujeres, concluyendo que existe una baja presencia de mujeres en cargos de alta dirección universitaria (Del Pino Arriagada et al., 2018).

Los autores Christopher Martínez y Juan Carlos Arellano en un estudio reciente de este año, establecen que la brecha de género en las rectorías de universidades chilenas es abismante, esto debido a que las universidades chilenas han sido gobernadas por mujeres solo el 3,3\% del tiempo entre 1990 y 2019 (Martínez, Arellano, 2021).

\subsection{Elección de autoridades}

Dentro de esta investigación se ha decidido analizar los métodos de elección de los cargos de rector, vicerrector y decano dentro de las universidades chilenas estatales y privadas, en el contexto de su marco legal vigente, como parte de un marco teórico referencial.

I. Elección de Rector/a: se divide por universidades estatales y privadas:

a. Universidades Estatales: se encuentra regulado por la Ley 19.305 y 21.094.

i. Ley 19.305: establece que la elección del rector en universidades estatales debe ser mediante votación de los académicos de manera personal, secreta e informada, en donde el rector será elegido en votación directa y por mayoría absoluta de los votos válidamente emitidos. Además, define que pueden votar los académicos pertenecientes a las tres más altas jerarquías de la universidad que tengan, a lo menos, un año de antigüedad en la misma y también establece los requerimientos para ser elegido rector.

ii. Ley 21.094 sobre universidades estatales: establece que el Rector durará en su cargo cuatro años, pudiendo ser reelegido sólo una vez.

b. Universidades privadas: Cada universidad tiene autonomía para definir el método de elección del rector. En la mayoría de las universidades privadas el rector es designado por la junta directiva o consejo académico correspondiente.

i. Universidades católicas: En estas universidades el rector es nombrado por la competente autoridad eclesiástica, la cual puede ser el arzobispo u obispo de la zona según corresponda, quien elige al rector de una terna confeccionada por un comité de búsqueda designado por los directivos de la universidad. El rector elegido debe ser ratificado por la Santa Sede. El rector en estas universidades dura cinco años en su cargo.

II. Elección de vicerrector/a: En todas las universidades los vicerrectores son nombrados por la junta directiva, previa propuesta del rector, debido a que es un cargo de exclusiva confianza del rector.

III. Elección de decano/a: Cada universidad tiene autonomía para definir el método de elección del decano. En la mayoría de universidades, el decano es elegido mediante votación de los académicos de la planta regular, profesores titulares y asociados, con 
un requisito de tiempo de permanencia en la universidad que está entre los dos y tres años.

Dentro de este proceso en gran parte de las universidades privadas un comité de búsqueda de la Facultad, es encargado de elaborar una terna de candidatos, la cual debe ser validada por el rector. Luego los académicos titulares y asociados eligen al decano por medio de elecciones. La duración del cargo de decano varía entre los dos y los cinco años según la universidad.

\section{Metodología}

El objetivo general de esta investigación fue analizar los factores que promueven o limitan la participación laboral femenina en la gestión directiva universitaria en Chile, para lo anterior, se establecieron tres objetivos específicos: a) Identificar la participación femenina en cargos directivos unipersonales universitarios en Chile: rectorías, vicerrectorías y decanatos, b) Identificar factores explicativos, para entender la participación femenina en cargos directivos, desde la perspectiva de actores claves y c) Establecer desafíos centrales para enfrentar los niveles de participación laboral femenina en cargos de gestión directiva universitaria en Chile.

El diseño fue no experimental, transversal y descriptivo de la situación encontrada en la gestión directiva universitaria en Chile. Se analizaron los cargos de rectoría, vicerrectoría y decanatos. Se han escogido estos cargos debido a que son los cargos de carácter unipersonal con jerarquía más alta al que puede acceder un académico/a dentro de una universidad. La investigación posee un enfoque mixto en la recolección de datos. Se utiliza el método cuantitativo para estudiar la presencia de mujeres en la dirección universitaria y simultáneamente se utiliza el método cualitativo para estudiar y analizar las razones que explican la baja presencia de mujeres en estos cargos. Para la recolección de datos, se estableció un método de recolección de datos a cada objetivo específico de la investigación variando entre análisis de datos y entrevistas semiestructuradas.

El análisis documental consideró la recolección de información concerniente a la cantidad de mujeres que poseen los cargos de Rector, Vicerrector y Decano, en las 54 universidades chilenas, a través de la información oficial que estas presentan en sus sitios web. La información se recolectó durante el primer semestre del año 2021, hasta julio de 2021. Esta información se tabuló en Excel y se dividió según las variables de universidades estatales y privadas.

Las entrevistas fueron aplicadas a once entrevistas a informantes claves. Se entrevistó a cuatro vicerrectoras, a seis decanas y a una experta en el tema, perteneciente al departamento de la equidad de género del CRUCH. Estas entrevistas se realizaron vía online, por la plataforma Zoom, dentro del contexto de pandemia mundial de coronavirus del año 2020/2021. Para la realización de estas entrevistas se entregó un consentimiento informado a cada entrevistada, en donde se señala que su participación es de carácter voluntario, la información recolectada es de carácter secreto y solo será utilizada con fines académicos para la presente investigación.

\section{Resultados}

\subsection{Presencia de mujeres en rectoría, vicerrectoría y decanatos}

Se analizó la presencia de mujeres en los cargos de rectoría, vicerrectoría y decanatos de todas las universidades de Chile, a través de información institucional en 
sus páginas web. Se analizó un total de 54 universidades chilenas que se dividen en 18 estatales y 36 privadas. Estas son:

Tabla 1: Universidades Estatales:

\begin{tabular}{|l|l|r|l|}
\hline \multicolumn{3}{|l|}{ Universidades Estatales: } \\
\hline 1 & Universidad de Tarapacá & 10 & Universidad de Chile \\
\hline 2 & Universidad Arturo Prat & 11 & Universidad Metropolitana de Ciencias de la Educación \\
\hline 3 & Universidad de Antofagasta & 12 & Universidad de O’Higgins \\
\hline 4 & Universidad de Atacama & 13 & Universidad de Talca \\
\hline 5 & Universidad de la Serena & 14 & Universidad del Bío-Bío \\
\hline 6 & Universidad de Playa Ancha & 15 & Universidad de la Frontera \\
\hline 7 & Universidad de Valparaíso & 16 & Universidad de Aysén \\
\hline 8 & Universidad de Santiago de Chile & 17 & Universidad de Magallanes \\
\hline 9 & Universidad Tecnológica Metropolitana & 18 & Universidad de Los Lagos \\
\hline
\end{tabular}

Fuente: elaboración propia en base a información del Consorcio de Universidades del Estado de Chile (CUECH).

Tabla 2: Universidades Privadas:

Universidades Privadas:

\begin{tabular}{|l|l|l|l|}
\hline 1 & Pontificia Universidad Católica de Chile & 18 & Universidad Finis Terrae \\
\hline 2 & Pontificia Universidad Católica de Valparaíso & 19 & Universidad Católica Silva Henríquez \\
\hline 3 & Universidad Católica de la Santísima Concepción & 20 & Universidad Santo Tomas \\
\hline 4 & Universidad Católica de Temuco & 21 & Universidad de las Américas \\
\hline 5 & Universidad Católica del Maule & 22 & Universidad SEK \\
\hline 6 & Universidad de Concepción & 23 & Universidad UNIACC \\
\hline 7 & Universidad de los Andes & 24 & Universidad Adventista de Chile \\
\hline 8 & Universidad Diego Portales & 25 & Universidad Pedro de Valdivia \\
\hline 9 & Universidad Alberto Hurtado & 26 & Universidad de Antofagasta \\
\hline 10 & Universidad Andrés Bello & 27 & Universidad Austral de Chile \\
\hline 11 & Universidad del Desarrollo & 28 & Universidad Católica del Norte \\
\hline 12 & Universidad Adolfo Ibáñez & 29 & Universidad Central de Chile \\
\hline 13 & Universidad Autónoma de Chile & 30 & Universidad Técnica Federico Santa María \\
\hline 14 & Universidad San Sebastián & 31 & Universidad de Aconcagua \\
\hline 15 & Universidad Mayor & 32 & Universidad Miguel de Cervantes \\
\hline 16 & Universidad Bernardo O’Higgins & 33 & Universidad Bolivariana \\
\hline 17 & Universidad Academia de Humanismo Cristiano & 34 & Universidad Viña del Mar \\
\hline
\end{tabular}

Fuente: elaboración propia.

No se ha tomado en cuenta para esta investigación las siguientes universidades:

- Universidad Los Leones: debido a que pertenece al Instituto Profesional Los Leones y su organización es la de un Instituto Profesional.

- Universidades que cerraron o están en periodo de cierre: ARCIS, UCINF, Universidad Chileno-Británica de Cultura, Universidad La República.

En las rectorías se constató la presencia de un total de 48 rectores hombres, lo que equivale a un $88,88 \%$ y 6 rectoras mujeres, lo que corresponde a un $11,11 \%$.

Tabla 3: Resultados participación femenina en rectorías.

\begin{tabular}{|l|l|l|l|}
\hline Rectores/as & Estatal & Privada & Total \\
\hline Hombres & $15(83,33 \%)$ & $33(91,67 \%)$ & $48(88,88 \%)$ \\
\hline Mujeres & $3(16,66 \%)$ & $3(8,33 \%)$ & $6(11,11 \%)$ \\
\hline Total & 18 & 36 & 54 \\
\hline
\end{tabular}

Fuente: Elaboración propia. 
Las universidades con rectoras mujeres son: Universidad de Aysén, Universidad Santo Tomas, Universidad de las Américas, Universidad SEK, Universidad Tecnológica Metropolitana y la Universidad Metropolitana de Ciencias de la Educación.

El porcentaje de participación femenina en rectorías de $11,11 \%$ es más alto que lo constatado por otros autores. Esto se debe principalmente debido a que la Universidad Tecnológica Metropolitana y la Universidad Metropolitana de Ciencias de la Educación, eligieron a rectoras mujeres de manera democrática en el transcurso de esta investigación, específicamente en el mes de junio de 2021 y asumieron sus funciones en el mes de julio del mismo año.

Cabe destacar que existe un mayor porcentaje de rectoras mujeres en las universidades estatales $(16,66 \%)$ que en universidades privadas $(8,33 \%)$.

Además, la Universidad de Aysén es la primera universidad estatal con una rectora mujer elegida democráticamente y desde su fundación en 2015, solamente ha tenido rectoras mujeres (Roxana Pey 2015-2016, María Teresa Marshall 2016-2019, Natacha Pino 2019-Actualidad).

Respecto al cargo de vicerrectorías, se constató la presencia de un total de 234 vicerrectores/as, de los cuales 168 son hombres, lo que equivale a un 71,79\% y 66 son mujeres, lo cual corresponde a un $28,21 \%$.

Tabla 4: Resultados participación femenina en Vicerrectorías.

\begin{tabular}{|l|l|l|l|}
\hline Vicerrectores/as & Estatal & Privada & Total \\
\hline Hombres & $45(68,18 \%)$ & $123(73,21 \%)$ & $168(71,79 \%)$ \\
\hline Mujeres & $21(31,82 \%)$ & $45(26,79 \%)$ & $66(28,21 \%)$ \\
\hline Total & 66 & 168 & 234 \\
\hline
\end{tabular}

Fuente: Elaboración propia.

Cabe destacar que en el cargo de Vicerrectoría existe un porcentaje de vicerrectoras mujeres mayor en universidades Estatales $(31,82 \%)$ que en universidades privadas $(26,79 \%)$.

Se analizó la totalidad de cargos de vicerrectorías de todas las universidades investigadas y se contabilizó la cantidad de vicerrectoras que ejercen ese cargo. Las vicerrectorías se agruparon por finalidad.

Tabla 5: Participación femenina en Vicerrectorías por tipo de Vicerrectoría.

\begin{tabular}{|l|l|l|l|l|}
\hline Cargo & Hombres & Mujeres & Total & $\%$ Mujeres \\
\hline Vicerrectoría de Asuntos Internacionales & 0 & 1 & 1 & $100 \%$ \\
\hline Vicerrectoría de Personas & 0 & 2 & 2 & $100 \%$ \\
\hline Vicerrectoría de Pregrado & 0 & 3 & 3 & $100 \%$ \\
\hline Vicerrectoría de Vinculación con el Medio & 11 & 9 & 20 & $45 \%$ \\
\hline Vicerrectoría de Sede & 28 & 14 & 42 & $33,33 \%$ \\
\hline Vicerrectoría de Aseguramiento de Calidad & 7 & 3 & 10 & $30 \%$ \\
\hline Vicerrectoría Académica & 36 & 14 & 50 & $28 \%$ \\
\hline Vicerrectoría de Investigación & 24 & 7 & 31 & $22,58 \%$ \\
\hline Vicerrectoría de Desarrollo & 7 & 2 & 9 & $22,22 \%$ \\
\hline Vicerrectoría de Asuntos Estudiantiles & 7 & 2 & 9 & $22,22 \%$ \\
\hline Vicerrectoría de Adm. y Finanzas & 39 & 7 & 46 & $15,21 \%$ \\
\hline Vicerrectoría de Formación Integral & 2 & 0 & 2 & $0 \%$ \\
\hline Vicerrectoría de Tecnologías & 4 & 0 & 4 & $0 \%$ \\
\hline Vicerrectoría de Postgrado & 3 & 0 & 3 & $0 \%$ \\
\hline Total general & 168 & 66 & 234 & $28,21 \%$ \\
\hline
\end{tabular}

Fuente: Elaboración Propia.

La vicerrectoría de vinculación con el medio o también llamada en algunas universidades vicerrectoría de comunicaciones es la vicerrectoría con mayor participación de mujeres con un $45 \%$ de los cargos. 
Luego existen tres vicerrectorías con el $100 \%$ de participación femenina:

i. Vicerrectoría de pregrado: existe en solo tres universidades y en su totalidad es ocupada por mujeres.

ii. Vicerrectoría de asuntos internacionales: solo existe en la Pontificia Universidad Católica de Chile y es ejercida por una mujer.

iii. Vicerrectoría de personas: existe en solo dos universidades siendo ejercida en ambas por una mujer.

La vicerrectoría de administración y finanzas también llamada en algunas universidades como vicerrectoría económica, es la vicerrectoría con menor porcentaje de participación femenina con un $15,21 \%$.

Existen tres vicerrectorías que no poseen participación femenina:

i. Vicerrectoría de formación integral: Existe solamente en dos universidades en las cuales es ejercida únicamente por hombres.

ii. Vicerrectoría de tecnologías: existe en cuatro universidades en las cuales es ejercida solamente por hombres.

iii.Vicerrectoría de postgrado: existe solamente en tres universidades, en las cuales es ejercida solo por hombres.

En los cargos de decanatura se constató un total de 417 decanos/as, de los cuales se desagregan en un total de 307 decanos hombres, lo que equivale a un 73,62\% y un total de 110 decanas mujeres, que corresponde a un 26,38\%.

Cabe destacar que en algunas universidades no existe el cargo de decano de Facultad, el cual es reemplazado por el cargo de director/a de departamento o de escuela. Excepción: la Universidad Tecnológica de Chile INACAP no posee decanos, ni directores por lo que no fue considerada en este análisis por decanaturas.

Tabla 6: Resultados participación femenina en decanatos.

\begin{tabular}{|l|l|l|l|}
\hline Decanos/as & Estatal & Privada & Total \\
\hline Hombres & $104(80 \%)$ & $203(70,73 \%)$ & $307(73,62 \%)$ \\
\hline Mujeres & $26(20 \%)$ & $84(29,27 \%)$ & $110(26,38 \%)$ \\
\hline Total & 130 & 287 & 417 \\
\hline
\end{tabular}

Fuente: Elaboración propia.

Cabe destacar que en las decanaturas se constata un porcentaje de participación femenina mayor en las universidades privadas $(29,27 \%)$ que en universidades estatales $(20 \%)$.

Se dividió las facultades universitarias según la Clasificación Internacional Normalizada de la Educación 2013 (CINE), clasificación de la UNESCO que representa una clasificación de referencia que permite ordenar los programas educativos por niveles de educación y campos de estudio.

Con esta división se generó la Tabla 5 sobre participación femenina en decanaturas por tipo de decanaturas, que muestra las decanaturas de facultades con mayor y menor porcentaje de participación femenina en las universidades de Chile.

Tabla 7: Participación femenina en decanaturas por tipo de decanaturas.

\begin{tabular}{|l|l|l|l|l|}
\hline Tipo de Decanato & Hombres & Mujeres & Total & Porcentaje \\
\hline Educación & 19 & 17 & 36 & $47,22 \%$ \\
\hline Ciencias sociales, periodismo e información & 32 & 20 & 52 & $38,46 \%$ \\
\hline Salud y bienestar & 39 & 23 & 62 & $37,10 \%$ \\
\hline Artes y humanidades & 24 & 10 & 34 & $29,41 \%$ \\
\hline Servicios & 12 & 4 & 16 & $25 \%$ \\
\hline Ciencias naturales, matemáticas y estadística & 43 & 13 & 56 & $23,21 \%$ \\
\hline Agricultura, silvicultura, pesca y veterinaria & 15 & 4 & 19 & $21,05 \%$ \\
\hline
\end{tabular}




\begin{tabular}{|l|l|l|l|l|}
\hline Administración de empresas y derecho & 46 & 11 & 57 & $19,30 \%$ \\
\hline Ingeniería, industria y construcción & 73 & 8 & 81 & $9,88 \%$ \\
\hline Tecnologías de la información y la comunicación (TIC) & 4 & 0 & 4 & $0,00 \%$ \\
\hline Total general & 307 & 110 & 417 & $26,38 \%$ \\
\hline
\end{tabular}

Fuente: Elaboración propia.

Las facultades con más decanas mujeres son:

i. Educación $(47,22 \%)$.

ii. Ciencias sociales, periodismo e información $(38,46 \%)$.

iii. Salud y bienestar $(37,10 \%)$ : dentro de este grupo se encuentran las facultades de Salud, Medicina, Enfermería y Odontología.

Las facultades con menos decanas mujeres son:

i. Administración de empresas y derecho (19,30\%): dentro de este grupo se encuentran las facultades de Derecho, Economía, Negocios y Administración.

ii. Ingeniería, industria y construcción $(9,88 \%)$ : dentro de este grupo se encuentran las facultades de Ingeniería y Arquitectura.

iii. Tecnologías de la información y la comunicación (TIC) (0\%): solo se clasificó a cuatro facultades dentro de este grupo, las cuales en su totalidad cuentan con decanos hombres.

\subsection{Factores que explican la participación femenina en la gestión directiva} universitaria:

A continuación, se presentan los resultados obtenidos en las once entrevistas realizadas divididas en las cuatro unidades de análisis, anteriormente mencionadas.

Sobre la percepción de la participación femenina en cargos directivos universitarios en la actualidad, todas las entrevistadas comparten la percepción que la participación femenina es menor a la participación masculina en cargos directivos universitarios y que la participación femenina en cargos directivos universitarios es baja. Además, todas las entrevistadas comparten que las mujeres poseen las mismas capacidades que los hombres, por lo cual no sería un factor explicativo de la baja presencia de mujeres en cargos directivos universitarios, sin embargo, varias entrevistadas sostienen que las mujeres mayoritariamente no llegan al grado de doctorado por distintos motivos, siendo la maternidad uno de los principales.

Al realizar la pregunta ¿Cuál es la mayor dificultad que ha tenido al asumir su cargo? Las respuestas obtenidas se pueden dividir en cuatro grupos:

a) Rechazo masculino: un total de cinco entrevistadas sostienen que las mayores dificultades se deben al rechazo masculino principalmente del liderazgo femenino.

El liderazgo femenino se pone en duda de manera constante, generando que las mujeres tengan que reafirmar sus convicciones y decisiones lo cual genera frustración y cansancio. Esto se ve reflejado en la respuesta de una vicerrectora de universidad estatal que establece que "El problema al cual yo me he enfrentado, pero que es una forma de percepción, es la oposición, el rechazo del mandato de una mujer. Eso es una situación que yo he podido percibir, dentro del contexto de esta mayor sensibilidad que tenemos las mujeres para percibir verdad todo lo que tiene que ver un poco con la conducta de las personas" (Vicerrectora de una universidad estatal).

Dentro de este tópico se resalta la dificultad que observan varias entrevistadas sobre la notoria falta de mujeres en los cuerpos colegiados, los cuales en su mayoría son ocupados por hombres, lo que, según tres entrevistadas, han experimentado situaciones de exclusión dentro de estos cuerpos colegiados. Esto se evidencia en la respuesta de una 
decana de universidad privada que responde que "Sí, percibo una dificultad cuando me enfrento a organismos colegiados entre pares, porque allí no es normal que exista un gran número de decanas, vicerrectoras, entonces eso hace que ahí se perciba una diferencia" (Decana de una universidad privada).

b) Vida familiar: tres entrevistadas establecen que la principal barrera es conciliar la vida familiar con la vida laboral, debido a que la vida familiar es la que más se resiente al asumir el cargo, debido al tiempo que requiere el cargo. Dentro de este tópico, se realizó la pregunta a todas las entrevistadas de que si la vida familiar es una barrera al ejercer el cargo en el cual se desempeñan:

- En su mayoría las entrevistadas respondieron que la vida familiar no es una barrera para ellas, debido a diversas razones. Algunas de estas razones son: que poseen apoyo familiar, distribución equitativa de las tareas domésticas y de los roles dentro del hogar con el marido/pareja o por no tener hijos.

- Sin embargo, todas las entrevistadas reconocen que la vida familiar es una barrera para las mujeres en su mayoría, que han visto a otras mujeres no poder avanzar debido a problemas familiares y que es muy importante poseer apoyo en el hogar y equilibrio en los roles familiares y domésticos dentro del hogar.

Este hallazgo es importante para la investigación, debido a que demuestra que la mayoría de las entrevistadas (quienes han logrado llegar al cargo de vicerrectora o decana) han podido superar esta barrera.

c) Naturaleza de las actividades académicas: Gestión vs Investigación: esta barrera no es nombrada inicialmente por las entrevistadas, sin embargo, seis entrevistadas establecen que existen dificultades para afrontar la gestión y la investigación al mismo tiempo, debido al tiempo que significan ambas labores académicas. Además, tres entrevistadas establecen que la investigación posee más valor o peso que la gestión dentro de la carrera académica universitaria, para alcanzar la jerarquía de profesor titular. Por ende, las mujeres prefieren asumir tareas de investigación en vez de gestión. Esto es revelado por las declaraciones de una decana de universidad privada: "Ocurre que en este proceso no siempre se valora de la misma manera los cargos de gestión, en el sentido de que tienden a prevalecer otro tipo de indicadores, fundamentalmente las publicaciones. La investigación pesa bastante y estas tareas de gestión consumen mucho tiempo que entonces no permite mantener un nivel de productividad científica, como la que se puede tener sin tener que destinar energías a un cargo de gestión. Entonces, en ese sentido, la valoración de estos cargos y su efecto en la carrera académica creo que es un tema pendiente..." (Decana de universidad privada).

Además, la maternidad femenina incide en esta barrera debido a que significa meses sin productividad científica, lo que genera que se prefiera aprovechar el tiempo en tareas académicas con mayor valor como la investigación. Esto lo demuestra la respuesta de una decana de universidad privada "...es evidente que el ámbito del cuidado de los hijos pequeños, todo el periodo que se alargan los pre y post natales de todas las mujeres, es una dificultad en todas las colaboradoras y académicas que uno tiene. Finalmente es un año y medio lo que están fuera, eso detiene su carrera académica, es una realidad empírica que veo acá" (Decana de universidad privada).

d) Dificultades propias del cargo: un total de tres entrevistadas sostienen que no han tenido mayores dificultades por ser mujer y que las principales dificultades son de gestión propias del cargo el cual están ejerciendo. Además, se menciona a la pandemia de coronavirus del año 2020/2021 como una dificultad que ha significado un desafío al cual se han tenido que adaptar rápidamente.

\section{3 Percepción sobre las oportunidades de asumir cargos directivos.}


Todas las entrevistadas establecieron que en la actualidad existen mejores oportunidades para que las mujeres asuman este tipo de cargos, principalmente debido a la visibilización del rol de la mujer y a la existencia de mayores espacios de inclusión de la mujer en las universidades. Esto lo demuestra la respuesta de una decana de universidad privada, a la pregunta de si existen mejores oportunidades para que la mujer asuma este tipo de cargos en la actualidad: “...de todas maneras, te podría decir que hace 20 o 30 años atrás era mucho más complejo para una mujer, primero por la visibilización que existe hoy día a nivel cultural sobre la necesidad de que las mujeres se inserten en el mundo del trabajo y que asuman cargos de relevancia. Hay una visión más amplia y socialmente también hay una mayor visibilización del rol de la mujer" (Decana de universidad privada).

Además, se establece que ha existido un cambio en los últimos cinco años en el cual el enfoque está puesto más en el desempeño, que en el género.

Dentro de este grupo, se realizó la pregunta sobre si creen que el cambio que ha existido con respecto a una mayor inserción femenina en estos cargos, se ha producido debido a un cambio generacional o a un cambio cultural. Un total de ocho entrevistadas sugieren que este fenómeno se debe a ambos cambios, en la cultura y generacional, mientras que tres entrevistadas sugieren que el cambio generacional trae consigo el cambio cultural.

Dentro de las acciones a implementar para promover la participación femenina en cargos directivos universitarios, las respuestas son variadas y se pueden dividir en tres grupos:

a) Ajuste de normativas: Con respecto a ajustar las normativas y estatutos de las universidades, seis entrevistadas establecen que se deben ajustar las normativas para implementar el enfoque de género en todas las áreas de las universidades. De esta manera tomar en consideración la realidad de las mujeres e incorporar mayor flexibilidad en temas familiares como la maternidad, principalmente en el desarrollo de la carrera académica universitaria. Esto se ve reflejado en lo expuesto por una vicerrectora de universidad privada "...la reglamentación debe incluir el enfoque de género porque no podemos realizar esas actividades de la misma forma que los varones. No implica que no tengamos la capacidad, pero la manera en que la hacemos puede ser distinta. Por ejemplo, si yo le voy a dar a un varón un año para que llegue a una capacitación, a una mujer le puedo dar un año y medio, porque eventualmente si se encuentra en edad fértil podría quedar embarazada o algún otro evento natural propio del género" (Vicerrectora de universidad privada).

Dentro de este mismo tema, tres entrevistadas sugieren generar un equilibrio entre la valoración que se le da a las tareas de gestión vs las tareas de investigación dentro de la carrera académica. De esta manera podría existir mayor interés por ejercer cargos de gestión.

Con respecto al enfoque de género, en la actualidad varias universidades principalmente estatales, están en proceso de cambio de sus estatutos y es muy importante que lo incluyan en sus normativas, para promover la participación femenina en los cargos directivos. Además, es necesario establecer que algunas universidades ya han implementado el enfoque de género en sus normativas.

b) Cuotas de género: Se preguntó a las entrevistadas su opinión sobre imponer cuotas de género en los cargos directivos universitarios:

- Solamente cuatro entrevistadas responden que deben implementarse en un primer momento de manera razonable y no impuesta, para que la mujer ingrese a espacios donde ha sido más difícil su ingreso. El principal argumento a favor de las cuotas de género 
establece que deben ser aplicadas de manera razonable, en el sentido de no imponer cuotas que sean muy difíciles o imposibles de implementar. Una entrevistada expone como ejemplo que, en la carrera de metalurgia en minas, se están imponiendo cuotas de género de casi un 50\% para mujeres, cuando no hay 50\% de mujeres que estudien esa carrera universitaria. Por lo cual, si se implementan, éstas deberían ser acordes al contexto y a la realidad que posee el área en específico.

Otro argumento a favor de las cuotas de género establece que éstas deberían imponerse en un comienzo y que luego de un periodo de tiempo determinado (dos procesos eleccionarios) se retiren estas cuotas de género, de manera que sea un impulso para la participación femenina en los cargos directivos universitarios.

Mientras que otro argumento establece que las cuotas de género deben implementarse solamente en los casos en que el poder no se ceda y sea la única forma de que la mujer pueda ingresar a ciertos cargos directivos.

- Siete entrevistadas establecen que no deberían imponerse cuotas de género, debido a que se busca a los mejores para estos cargos directivos independientemente de su género y que el método de elección debiera ser objetivo.

Una vicerrectora entrevistada dice que posee tres direcciones a su cargo, las cuales las tres están bajo la dirección de una mujer. Ella expone que, si tuviera que implementar cuotas de género tendría que sacar a una mujer de una dirección y poner a un hombre, cuando ella eligió (por concurso público) a las tres directoras porque eran las mejores para ese cargo.

Por ende, se argumenta que las cuotas de género son discriminatorias y que además limitan la elección de estos cargos directivos.

Además, otro argumento establece que puede ser hasta ofensivo para algunas personas el hecho de elegir a alguien solamente por su género y no por sus capacidades o desempeño.

Otro argumento establece que sería difícil o imposible imponer cuotas de género en una institución privada.

c) Visibilización y formación: Dentro de las respuestas obtenidas, existen conceptos que se repiten:

a. Visibilización y promoción de la labor de las mujeres académicas dentro de la universidad: tres entrevistadas sugieren que, si se conoce la labor y tarea de mujeres académicas dentro de la universidad, existen más posibilidades que pueden ser elegidas en cargos de elección popular como rectora o decana, y como vicerrectora, ya que se necesita del conocimiento y confianza del rector.

La confianza también es destacada como un concepto interesante principalmente para el cargo de vicerrectoría, debido a que se necesita del conocimiento y principalmente de la confianza del rector, para ejercer el cargo.

Esto se ve reflejado en lo expuesto por una vicerrectora de universidad estatal: "yo creo que esto es una cosa de confianza y que nos conozcan porque si yo estoy en este cargo es porque el rector conocía mis competencias, conocía mis capacidades también mis limitaciones y él pensó que yo podría estar en la vicerrectoría. Pero porque me conocían, entonces en la medida de que uno conoce, uno confía, si uno confía entonces participa" (Vicerrectora de universidad estatal)

b. Formación: tres entrevistadas establecen que es necesario contar con procesos de formación que promuevan el desarrollo de competencias de liderazgo. Una de las entrevistadas sugiere una escuela de líderes, como una instancia de formación sobre habilidades de planificación, administración de personas y principalmente habilidades de liderazgo de manera de asumir una posición de liderazgo en el rol a ejercer. Esto se ve reflejado en los descrito por una decana de universidad privada: "La primera acción es la 
formación. En la universidad podríamos promover mayores programas de liderazgo, mayores programas que tengan que ver en cómo administrar mejores recursos humanos, yo creo que más que nada liderazgo, como asumir una posición de liderazgo en el rol que te toque" (Decana de universidad privada)

\section{Conclusiones}

Se puede concluir que la participación femenina es baja en comparación a la participación masculina, siendo el cargo con mayor diferencia el cargo de rector. No obstante, se ve optimista y promisorio el porcentaje de $11,11 \%$ de participación femenina en rectoría, que es más alto que lo establecido por otros autores en estudios pasados. También se puede concluir que porcentualmente está muy igualada la participación femenina en los cargos de vicerrectoría $(28,21 \%)$ y decanato $(26,38 \%)$, ambos muy cercanos al 27\%, sin embargo, existen diferencias cuando se distingue entre universidades estatales y privadas, existiendo mayor porcentaje de participación femenina en las vicerrectorías de universidades estatales $(31,82 \%)$ que en vicerrectorías de universidades privadas $(26,79 \%)$, al revés de lo que pasa en los decanatos donde hay mayor porcentaje de participación femenina en universidades privadas $(29,27 \%)$ que en universidades estatales (20\%). Con respecto a la diferenciación por tipo, se puede concluir que existe mayor participación femenina en los cargos de vicerrector y decano, en las ciencias de carácter más social y en aquellas labores o áreas que requieren vinculación con personas como la salud, educación o la vicerrectoría de vinculación con el medio. Por otra parte, existe menor participación femenina en aquellas labores de carácter más técnico, como administración de empresas, ingeniería, tecnologías, vicerrectoría de administración y finanzas.

Respecto a los factores que explican la participación femenina en la gestión directiva universitaria, se puede concluir a través del análisis efectuado a las entrevistas, que las principales barreras encontradas son:

1) Rechazo masculino: las respuestas obtenidas por algunas entrevistadas, da cuenta de la existencia de una percepción femenina de que existe un rechazo masculino, principalmente al liderazgo femenino.

2) Vida familiar: se concluye que es necesario un equilibrio de los roles familiares en el hogar, entre marido/padre y esposa/madre.

3) Naturaleza de las actividades académicas: gestión vs investigación: dificultad para ejercer labores de gestión e investigación al mismo tiempo, debido al tiempo que conllevan. Además de la existencia de una percepción de que las tareas y los cargos de gestión poseen menor valor dentro de la carrera académica universitaria.

Finalmente se destacan como desafíos en la temática:

1) Promover una valoración equilibrada entre las tareas y cargos de gestión versus las tareas de investigación, dentro de la carrera académica universitaria. Es importante generar una mejor valoración a las tareas y los cargos de gestión debido a la importancia que también poseen.

De esta manera podría existir mayor interés por ejercer cargos de gestión, ya que estos tendrían una mejor valoración para ascender y avanzar dentro de la carrera académica universitaria.

2) Implementar la perspectiva y enfoque de género como una política institucional, dentro de los estatutos o reglamentos, principalmente en el área de desarrollo de la carrera académica universitaria. Es importante la inclusión de la perspectiva de género de manera de incorporar mayor flexibilidad principalmente en la incidencia de la maternidad en el desarrollo de la carrera académica. 
3) Se destaca como último desafío la implementación de cuotas de género, esto debido a la controversia que su aplicación genera y debido al contraste de los argumentos y respuestas obtenidas, con entrevistadas que están a favor y otras en contra.

Esta investigación concluye que es una acción que posee opiniones muy divididas y contrarias entre las entrevistadas, generando adeptos y opositores, debido a que estos cargos deben ser ocupados por personas con las mejores competencias correspondientes para el cargo. Por lo tanto, su aplicación debe ser con una aceptación unánime, con porcentajes de participación acordes al contexto en donde se aplican y en áreas en que la participación femenina sea muy escasa y, además, constituyan un difícil acceso para las mujeres.

\section{Referencias}

Astelarra Judith, (2005) El sistema de género, nuevos conceptos y metodología. Depto. de Sociología Universidad Autónoma de Barcelona, Centro de Estudios Miguel Henríquez (CEME).

https://www.archivochile.com/Mov_sociales/mov_mujeres/doc_gen_cl/MSdocgenc1001 4.pdf

Barberá, E. (1998). Psicología y género. I. M. Benlloch (Ed.). Barcelona: Ariel.

Bakan, D. (1966), "The Duality of Human Existence." Boston: Beacon Press.

Becher, Tony (2001), Tribus y territorios académicos. La indagación intelectual y las culturas de las disciplinas, Barcelona, Gedisa.

Bedia, R. C. (2005). El género en las ciencias sociales. Cuadernos de trabajo social, 18, 249-258.

https://revistas.ucm.es/index.php/CUTS/article/download/CUTS0505110249A/7595

Berríos, P. (2007). Análisis sobre los profesores universitarios y desafíos para la profesión académica en Chile. Revista Calidad de la Educación Superior, 26, 39-53 https://www.calidadenlaeducacion.cl/index.php/rce/article/view/232/235

Bourdieu, P. (2008). Homo Academicus. 1a. Edición Español. Buenos Aires: Siglo XXI Editores.

Cárdenas, A., Correa, N., \& Prado, X. (2014). Segregación laboral y género: tendencias y desafíos relativos al mercado laboral de la salud y la educación en Chile. Polis. Revista Latinoamericana, (38). https://journals.openedition.org/polis/10182

CIRILLO, Lidia 2005 «Virtualidades pedagógicas del feminismo para la izquierda», en Revista Internacional de Filosofía Política (UNED-Madrid/UAM-México), no 25, 2005

Cuadrado, I. (2004). Valores y rasgos estereotípicos de género de mujeres líderes. Psicothema, 16(2), 270-275. https://www.redalyc.org/pdf/727/72716215.pdf

Del Pino Arriagada, S., Vallejos Cartes, R., Améstica Rivas, L., \& Cornejo-Saavedra, E. (2018). Presencia de las mujeres en la alta gestión universitaria. Las universidades públicas en Chile. Páginas De Educación, 11(2), 176-198. https://revistas.ucu.edu.uy/index.php/paginasdeeducacion/article/view/1633/1611

Eagly, A. (1987). Sex differences in social behavior: A social-role interpretation. Hillsdale. Lawrence Erlbaum Associates

Gaete-Quezada, Ricardo (2015), "El techo de cristal en las universidades estatales chilenas. Un análisis exploratorio", en Revista Iberoamericana de Educación Superior (ries), México, unam-iisue/Universia, vol. vi, núm. 17, pp. 3-20. http://www.scielo.org.mx/scielo.php?pid=S2007-

$28722015000300003 \&$ script=sci_arttext

Glass Ceiling Commission. (1995). Glass Ceiling Commission-Good for business: Making full use of the nation's human capital. 
https://ecommons.cornell.edu/bitstream/handle/1813/79348/GlassCeilingFactFind ingEnvironmentalScan.pdf? sequence $=1$ \&isAllowed $=\mathrm{y}$

Guil, A. (2007), "Docentes e investigadoras en las universidades españolas: visualizando techos de cristal", en Revista de Investigación Educativa, vol. 25, núm. 1, pp. 111131. https://revistas.um.es/rie/article/view/96561/92781

Hymowitz, C. y Schelhardt, T. (1986). "Corporate women: special report." Wall Street Journal, march 24.

Instituto Nacional de Estadísticas. (2021). Boletín Estadístico: empleo trimestral, Edición $\mathrm{n}^{\circ} 270$. Ministerio de Economía. Chile.

Lamas, M. (1986), La antropología feminista y la categoría "género" en Nueva Antropología, vol. VIII, 173-198. Asociación Nueva Antropología A.C. Distrito Federal, México. https://www.redalyc.org/pdf/159/15903009.pdf

Lorenzo Delgado, M., Sola Martínez, T., \& Cáceres Reche, M. P. (2007). Principales referentes legales y educativos para el desarrollo de la coeducación. https://digibug.ugr.es/bitstream/handle/10481/28575/564.\%20n.\%2037.pdf?seque $\underline{\text { nce }=1}$

Lupano, M.L., \& Castro, A. (2010). Análisis de características estereotípicas de género. https://www.redalyc.org/pdf/4595/459545418002.pdf

Martínez, C.A., Arellano, (2021). J.C. University presidents' turnover and survival: the case of Chile. High Educ. https://www.ciperchile.cl/2021/03/31/escaner-a-losrectores-chilenos-como-limitar-su-poder/

Mead, M. (1972). Educación y cultura, término género. (3ra. Ed.) Buenos Aires, Argentina. Editorial Paidos.

Merton, R. (1968), "The Matthew effect in science. The reward and communication systems of science are considered", en Science, 159 (3810), pp. 56-63. https://www.science.org/doi/10.1126/science.159.3810.56

Mujeres Empresarias y DESUC. (2021). Informe de Mujeres en Alta Dirección de Empresas (IMAD 2020). Empresas Grandes y Medianas. Fyrma.

Organización de las Naciones Unidas para la Educación, la Ciencia y la Cultura [UNESCO]. (2021). Mujeres en la educación superior: ¿la ventaja femenina ha puesto fin a las desigualdades de género?

https://www.iesalc.unesco.org/wp-content/uploads/2021/03/Las-mujeres-en-laeducación-superior_12-03-21.pdf

Parsons, T., \& Bales, R.G. (1955). Family, socialization and interaction process. Glencoe: Free Press.

Programa de las Naciones Unidas para el Desarrollo (PNUD). (2016). Igualdad de género: ¿por qué es importante? https://www.un.org/sustainabledevelopment/es/wpcontent/uploads/sites/3/2016/10/5 Spanish Why it Matters.pdf

Rossiter MW (1993) The Matthew Matilda effect in science. Social Studies of Science 23:325-341. https://journals.sagepub.com/doi/abs/10.1177/030631293023002004

UNESCO-UIS (2013) Clasificación Internacional Normalizada de la Educación CINE 2011. Instituto de Estadística de la UNESCO (UIS). Montreal. https://www.consejoderectores.cl/wpcontent/uploads/anuario/2013/Clasificacion_Internacional_Normalizada_de_la_E ducacion CINE-UNESCO.pdf 